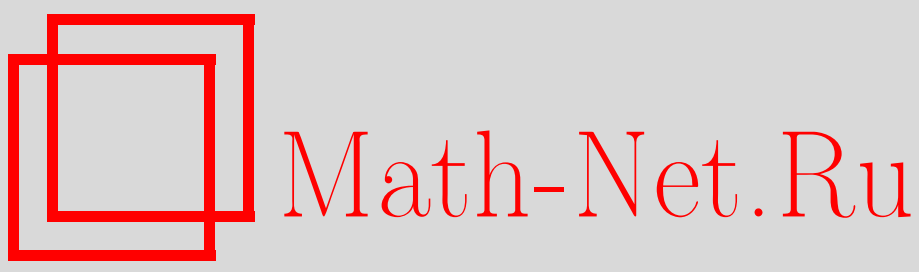

А. Ю. Колесов, Н. Х. Розов, Параметрическое возбуждение высокомодовых колебаний у нелинейного телеграфного уравнения, Матем. сб., 2000, том 191, номер 8, 45-68

DOI: https://doi.org/10.4213/sm498

Использование Общероссийского математического портала Math-Net.Ru подразумевает, что вы прочитали и согласны с пользовательским соглашением

http://www.mathnet.ru/rus/agreement

Параметры загрузки:

IP : 54.198 .55 .26

26 апреля 2023 г., 11:45:02 


\title{
Параметрическое возбуждение высокомодовых колебаний у нелинейного телеграфного уравнения
}

\begin{abstract}
Проблема параметрического возбуждения высокомодовых колебаний решается для нелинейного телеграфоного уравнения с параметрическим внешним воздействием и малой диффузией, рассматриваемого (по пространственной переменной) на конечном отрезке и дополненного граничными условиями Неймана на его концах. Показано, что при подходящем выборе параметров внешнего воздействия у такой краевой задачи может существовать любое фиксированное число экспоненциально устойчивых решений, периодических по времени и быстро осциллирующих по пространственной переменной.

Библиография: 15 названий.
\end{abstract}

\section{Введение}

Физические основы параметрических колебаний представлены в классических работах Л.И. Мандельштама и Н. Д. Папалекси, а математические аспекты теории изложены в монографии [1], содержащей исследование нелинейного параметрического резонанса для обыкновенных дифференциальных уравнений. Для некоторых классов волновых уравнений формализм построения возбуждаемых параметрических колебаний предложен в [2]. Однако теория линейного параметрического резонанса продвинута столь далеко [3], [4], что позволяет придать результатам из [2] строгий смысл.

Изучение параметрического резонанса в сингулярно возмушенных системах по инициативе Ю. С. Колесова начато в заметке [5] на примере параболической системы типа реакция-диффузия с мальми коэффициентами диффузии. В дальнейшем эта тематика развивалась в работах [6]-[10], посвященных, главным образом, анализу параметрических колебаний в сингулярно возмущенных телеграфных уравнениях. Как отмечено Ю. С. Колесовьм [8], мотивировкой для таких постановок задач служит то обстоятельство, что здесь мы находимся в окрестности наиболее глубокой особенности. А это, в свою очередь, может позволить в полном объеме выявить все динамические эффекты, вызванные параметрическим внешним воздействием.

Плодотворность указанного подхода иллюстрируют в очередной раз и результаты настоящей статьи, посвященной нелинейному телеграфному уравнению с параметрическим внешним воздействием и малой диффузией. Показано сушествование у этого уравнения (при подходящем выборе параметров внешнего воздействия)

Работа вьполнена при поддержке Российского фонда фундаментальных исследований (грант № 96-01-00207).

(C) А.Ю. КолесОв, Н.Х. РОзов 2000 
любого фиксированного числа устойчивых решений, периодических по времени и быстро осциллирующих по пространственной переменной. Данный феномен, изучавшийся ранее в работе [10] и названный там параметрической буферностью, характерен именно для случая малой диффузии и в равной мере присуш как гиперболическим, так и параболическим уравнениям. Ниже предложена новая постановка задачи, в которой высокомодовый характер возбуждаемых параметрических колебаний проявляется наиболее ярко.

\section{§1. Сушествование высокомодовых периодических решений}

1.1. Постановка задачи и линейный анализ. На отрезке $0 \leqslant x \leqslant \pi$ рассмотрим краевую задачу

$$
\begin{gathered}
u_{t t}+\varepsilon u_{t}+(1+\varepsilon \alpha \cos 2 \omega t) u=\varepsilon^{2} a^{2} u_{x x}+f\left(u, u_{t}\right), \\
\left.u_{x}\right|_{x=0}=\left.u_{x}\right|_{x=\pi}=0
\end{gathered}
$$

где $0<\varepsilon \ll 1, a=\mathrm{const}>0$, а параметры $\alpha, \omega$, имеющие порядок единицы, таковы, что

$$
\omega>1, \quad \alpha>2 \omega .
$$

Относительно нелинейности $f(u, v) \in C^{\infty}$ будем предполагать, что ее тейлоровское разложение в нуле имеет вид

$$
f(u, v)=a_{1} u^{2}+a_{2} u v+a_{3} v^{2}+b_{1} u^{3}+b_{2} u^{2} v+b_{3} u v^{2}+b_{4} v^{3}+\cdots .
$$

В качестве фазового пространства (пространства начальных условий $\left(u, u_{t}\right)$ ) краевой задачи (1.1) возьмем $\stackrel{\circ}{W} \underset{2}{2}(0, \pi) \times W_{2}^{1}(0, \pi)$, где $\stackrel{\circ}{W} \underset{2}{2}-$ соболевское пространство функций, удовлетворяющих граничным условиям из (1.1). Напомним, что понятие фазового пространства позволяет практически без изменений перенести на бесконечномерный случай основные положения качественной теории обыкновенных дифференциальных уравнений: устойчивость по Ляпунову, теоремы об устойчивости по первому приближению, утверждения об интегральных многообразиях и т.д. Поэтому ниже для краевой задачи (1.1) соответствующие понятия используются без дополнительных пояснений.

В данной работе мы будем интересоваться вопросами о существовании и устойчивости $(2 \pi / \omega)$-периодических по $t$ решений краевой задачи $(1.1)$, бифурцирующих при $\varepsilon>0$ из ее нулевого положения равновесия. В связи с этим сначала изучим свойства устойчивости последнего.

Отбросим в (1.1) нелинейность, а затем применим к получившейся линейной краевой задаче метод Фурье по системе функций $\cos n x, n=0,1, \ldots$ В результате убедимся, что за устойчивость нулевого решения задачи (1.1) отвечает расположение мультипликаторов линейных уравнений

$$
\ddot{u}+\varepsilon \dot{u}+\left[\omega^{2}+\varepsilon \delta_{n}(\varepsilon)+\varepsilon \alpha \cos 2 \omega t\right] u=0, \quad n=0,1, \ldots,
$$

где точка - дифференцирование по $t$, a

$$
\delta_{n}(\varepsilon)=\frac{1-\omega^{2}+\varepsilon^{2} a^{2} n^{2}}{\varepsilon} .
$$


Заметим, что, как следует из результатов монографий [1] и [3], уравнения (1.4) могут иметь экспоненциально растущие решения только при тех $n$, для которых $\delta_{n}(\varepsilon) \sim 1$. Связано это с тем, что при $\delta_{n} \sim 1$ может наступить так называемый основной параметрический резонанс, а при “отходе" от него, т.е. при увеличении $\left|\delta_{n}\right|$, нулевое решение уравнений (1.4) становится экспоненциально устойчивым. Отметим еше, что принципиальная возможность выполнения при некоторых $n$ условия $\delta_{n}(\varepsilon) \sim 1$ вытекает из первого неравенства (1.2) и определения (1.5).

Все вышесказанное наводит на мысль о целесообразности рассмотрения вместо (1.4) вспомогательного уравнения

$$
\ddot{u}+\varepsilon \dot{u}+\left[\omega^{2}+\varepsilon \delta+\varepsilon \alpha \cos 2 \omega t\right] u=0
$$

в котором параметр $\delta$ непрерьвно меняется на некотором конечном отрезке. Свойства устойчивости подобных уравнений хорошо изучены (см. [1], [3]). Однако наиболее просто они выявляются с помощю алгоритма из [11], суть которого в следующем.

Подставим в (1.6) выражение

$$
u=V(t, \varepsilon, \delta) \exp [\varepsilon D(\varepsilon, \delta) t]
$$

где $(2 \pi / \omega)$-периодическая по $t$ матрица-строка $V=[v(t, \varepsilon, \delta), \bar{v}(t, \varepsilon, \delta)]$ и квадратная матрица

$$
D(\varepsilon, \delta)=\left(\begin{array}{ll}
d_{1}(\varepsilon, \delta) & d_{2}(\varepsilon, \delta) \\
\bar{d}_{2}(\varepsilon, \delta) & \bar{d}_{1}(\varepsilon, \delta)
\end{array}\right)
$$

с комплексными элементами таковы, что

$$
\begin{aligned}
v(t, \varepsilon, \delta) & =\exp (i \omega t)+\varepsilon v_{1}(t, \delta)+\cdots \\
d_{j}(\varepsilon, \delta) & =d_{j}^{0}(\delta)+\varepsilon d_{j}^{1}(\delta)+\cdots, \quad j=1,2
\end{aligned}
$$

Приравнивая, далее, коэффициенты при $\varepsilon$, для определения $v_{1}(t, \delta)$ получаем линейное неоднородное уравнение, а из условий его разрешимости в классе $(2 \pi / \omega)$-периодических функций находим элементы $d_{j}^{0}(\delta), j=1,2$, матрицы $D(0, \delta)$. Опуская несложные вычисления, приведем сразу окончательный результат:

$$
d_{1}^{0}=-\frac{1}{2}+i \delta(2 \omega)^{-1}, \quad d_{2}^{0}=i \alpha(4 \omega)^{-1}
$$

Отметим еще, что, как показано в [12], фигурирующие в (1.7) матрицы можно выбрать аналитически зависящими от $\varepsilon$ и $\delta$, что и будем считать сделанньм.

Из (1.8) следует, что $\operatorname{tr} D(\varepsilon, \delta)<0$. Поэтому для того чтобы получить представление о свойствах устойчивости решений уравнения (1.6), а значит, и уравнений (1.4), обратимся к равенству

$$
\operatorname{det} D(\varepsilon, \delta)=0
$$

для нахождения критических значений параметра $\delta$. Привлекая в очередной раз (1.8) и учитывая второе неравенство (1.2), убеждаемся, что в точках $\varepsilon=0$, 
$\delta= \pm \sqrt{\alpha^{2} / 4-\omega^{2}}$ для уравнения (1.9) выполняются условия теоремы о неявной функции по переменной $\delta$. Тем самым однозначно определяются два его аналитически зависящие от $\varepsilon$ решения

$$
\delta=\delta_{j}^{*}(\varepsilon): \delta_{j}^{*}(0)=(-1)^{j} \delta_{*}, \quad \delta_{*}=\sqrt{\frac{1}{4} \alpha^{2}-\omega^{2}}, \quad j=1,2
$$

Подведем итог. Из очевидного неравенства $\operatorname{det} D(\varepsilon, \delta)<0$ при $\delta \in\left(\delta_{1}^{*}(\varepsilon), \delta_{2}^{*}(\varepsilon)\right)$ и из общих свойств линейных дифференциальных уравнений с периодическими коэффициентами (см., например, [3]) вытекает следующее утверждение.

ЛЕмма 1. Найдется такое не зависящие от $n$ достаточно малое число $\varepsilon_{0}>0$, что при $0<\varepsilon \leqslant \varepsilon_{0}$ имеем:

а) у всех уравнений (1.4) с номерами $n$, для которых $\delta_{n}(\varepsilon) \in\left(\delta_{1}^{*}(\varepsilon), \delta_{2}^{*}(\varepsilon)\right)$, есть әкспоненииально растущие решения;

б) при всех $n$, для которых $\delta_{n}(\varepsilon) \notin\left[\delta_{1}^{*}(\varepsilon), \delta_{2}^{*}(\varepsilon)\right]$, нулевое решение уравнений (1.4) экспоненциально устойчиво.

Итак, параметрический резонанс в краевой задаче (1.1) наступает только на конечном числе асимптотически высоких мод $\cos n x$ с номерами $n$, удовлетворяющими неравенствам

$$
\frac{1}{\varepsilon a} \sqrt{\omega^{2}-1+\varepsilon \delta_{1}^{*}(\varepsilon)}<n<\frac{1}{\varepsilon a} \sqrt{\omega^{2}-1+\varepsilon \delta_{2}^{*}(\varepsilon)} .
$$

Для того чтобы оценить количество $n_{*}(\varepsilon)$ таких номеров, учтем в $(1.11)$ равенства (1.10). В итоге убеждаемся, что

$$
n_{*}(\varepsilon) \sim \delta_{*}\left(a \sqrt{\omega^{2}-1}\right)^{-1}, \quad \varepsilon \rightarrow 0
$$

Из (1.12) следует, что количество возбужденных мод неограниченно увеличивается как при увеличении амплитуды внешнего воздействия $\alpha$, так и при $\omega \rightarrow 1$, $\varepsilon \rightarrow 0$. С физической точки зрения первое очевидно: увеличение энергетического параметра $\alpha$ должно приводить к повышению степени неустойчивости системы. Далее, при фиксированном $\alpha$ и при $\omega \rightarrow 1$ интервал (1.11) сдвигается в сторону все более низких мод, которые возбуждаются, естественно, легче, чем более высокие. Этим и объясняется неограниченное увеличение $n_{*}(\varepsilon)$ в данном случае. Например, при $\omega=1+\sqrt{\varepsilon} \Delta, \Delta=$ const $>0$, имеем $n_{*}(\varepsilon) \sim \varepsilon^{-1 / 4}$.

1.2. Принцип подобия. Для нахождения периодических решений задачи (1.1), бифурцирующих из нуля при $\varepsilon>0$ на модах с номерами (1.11), воспользуемся так называемым принципом подобия (см. [9], [13]). Его применение состоит из трех этапов.

Этап 1. Выполним в (1.1) замену переменной $n x \rightarrow x$, где $n$ - достаточно большое натуральное число. В результате придем к задаче

$$
\begin{gathered}
u_{t t}+\varepsilon u_{t}+(1+\varepsilon \alpha \cos 2 \omega t) u=\left(\sigma^{2}+\varepsilon \delta_{n}(\varepsilon)\right) u_{x x}+f\left(u, u_{t}\right), \\
\left.u_{x}\right|_{x=0}=\left.u_{x}\right|_{x=n \pi}=0,
\end{gathered}
$$


где $\sigma=\sqrt{\omega^{2}-1}$, а параметр $\delta_{n}$ (определенный в $\left.(1.5)\right)$ будем считать величиной порядка единицы.

Этап 2. Введем в рассмотрение вспомогательную задачу

$$
\begin{gathered}
u_{t t}+\varepsilon u_{t}+(1+\varepsilon \alpha \cos 2 \omega t) u=\left(\sigma^{2}+\varepsilon \delta\right) u_{x x}+f\left(u, u_{t}\right) \\
\left.u_{x}\right|_{x=0}=\left.u_{x}\right|_{x=\pi}=0
\end{gathered}
$$

получаюшуюся из (1.13) заменой дискретного параметра $\delta_{n}$ на непрерывный и переходом от отрезка $0 \leqslant x \leqslant n \pi$ к прежнему отрезку $0 \leqslant x \leqslant \pi$.

Предположим, далее, что на некотором промежутке

$$
\delta_{1} \leqslant \delta \leqslant \delta_{2}, \quad-\delta_{*}<\delta_{1}<\delta_{2}<\delta_{*}
$$

изменения $\delta$ она имеет $(2 \pi / \omega)$-периодическое по $t$ и нетривиально зависящее от $x$ решение

$$
u=u_{0}(t, x, \varepsilon, \delta)
$$

Продолжим это решение по $x$ на отрезок $-\pi \leqslant x \leqslant 0$ четным образом, а затем на всю ось $x$ по периодичности с периодом $2 \pi$. Тогда, как легко видеть, оно будет удовлетворять граничньм условиям из (1.13) при любом натуральном $n$, а при $\delta=\delta_{n}(\varepsilon) \in\left[\delta_{1}, \delta_{2}\right]-$ всей краевой задаче $(1.13)$.

Этап 3. Возвращаясь к исходной краевой задаче (1.1), заключаем, что при сделанных выше предположениях она имеет быстро осциллирующие по $x$ периодические решения

$$
u_{n}^{1}(t, x, \varepsilon)=u_{0}\left(t, n x, \varepsilon, \delta_{n}(\varepsilon)\right), \quad u_{n}^{2}(t, x, \varepsilon)=u_{0}\left(t, \pi-n x, \varepsilon, \delta_{n}(\varepsilon)\right)
$$

с номерами $n$, удовлетворяющими неравенствам

$$
\frac{1}{\varepsilon a} \sqrt{\omega^{2}-1+\varepsilon \delta_{1}} \leqslant n \leqslant \frac{1}{\varepsilon a} \sqrt{\omega^{2}-1+\varepsilon \delta_{2}}
$$

Сформулированњый принцип сводит интересующую нас проблему к исследованию вопроса о параметрическом возбуждении пространственно неоднородных периодических колебаний в рамках вспомогательной краевой задачи (1.14), регулярно зависяшей от всех своих параметров. Заметим, далее, что поскольку при $\varepsilon=0$ линеаризованная в нуле краевая задача (1.14) допускает тригонометрические решения

$$
\exp \left( \pm i \omega_{n} t\right) \cos n x, \quad \omega_{n}=\sqrt{1+\sigma^{2} n^{2}}, \quad n=0,1, \ldots
$$

а равенство $\omega_{n}=\omega$ выполняется, очевидно, лишь при $n=1$, то речь может идти только о ее периодическом решении, бифурцирующем из нуля при $\varepsilon>0$ на моде $\cos x$. Построение асимптотики этого решения, а также доказательство его существования базируются на известных асимптотических методах из [1], адаптированных для телеграфных уравнений в [6]-[8], [14]. Соответствующий анализ приводится ниже. 
1.3. Алгоритмическая часть. Для построения асимптотики упомянутого вьше периодического решения задачи (1.14) воспользуемся стандартным одночастотным методом [1]. Положим в (1.14)

$$
u=\sqrt{\varepsilon} u_{1}(t, x)+\varepsilon u_{2}(t, x)+\varepsilon^{3 / 2} u_{3}(t, x)+\varepsilon^{2} u_{4}(t, x)+\cdots,
$$

где

$$
u_{1}=[\xi \exp (i \omega t)+\bar{\xi} \exp (-i \omega t)] \cos x
$$

а $\xi$ - неизвестная (подлежашая определению) комплексная постоянная. Учитывая затем разложение (1.3) и приравнивая коэффициенты при одинаковых степенях $\varepsilon$, для определения $u_{k}, k=2,3,4$, получим рекуррентную последовательность линейных неоднородных краевых задач

$$
\left(\frac{\partial^{2}}{\partial t^{2}}-\sigma^{2} \frac{\partial^{2}}{\partial x^{2}}\right) u_{k}+u_{k}=g_{k}(t, x),\left.\quad \frac{\partial u_{k}}{\partial x}\right|_{x=0, \pi}=0
$$

Решения последних ишем в виде тригонометрических полиномов переменной $\omega t$ той же структуры, что и соответствующая неоднородность.

Несложные вычисления показывают, что

$$
u_{2}(t, x)=|\xi|^{2} A(x)+\xi^{2} B(x) \exp (2 i \omega t)+\bar{\xi}^{2} \bar{B}(x) \exp (-2 i \omega t)
$$

где

$$
\begin{aligned}
& A(x)=\left(a_{1}+\omega^{2} a_{3}\right)\left[1+\frac{1}{4 \omega^{2}-3} \cos 2 x\right] \\
& B(x)=\frac{1}{2}\left(a_{1}+i \omega a_{2}-\omega^{2} a_{3}\right)\left[\frac{1}{1-4 \omega^{2}}-\frac{1}{3} \cos 2 x\right]
\end{aligned}
$$

а при $k=3$ правая часть в (1.22) представляет собой линейную комбинацию функций

$$
\exp ( \pm i m \omega t) \cos p x, \quad m, p=1,3 .
$$

Поэтому для разрешимости задачи (1.22) при $k=3$ в нужном классе функций необходимо и достаточно отсутствие в правой части гармоник

$$
\exp ( \pm i \omega t) \cos x
$$

Учитьвая в представлении

$$
\begin{aligned}
g_{3}(t, x)= & -\frac{\partial u_{1}}{\partial t}-\alpha u_{1} \cos 2 \omega t+\delta \frac{\partial^{2} u_{1}}{\partial x^{2}}+2 a_{1} u_{1} u_{2}+a_{2} u_{1} \frac{\partial u_{2}}{\partial t}+a_{2} u_{2} \frac{\partial u_{1}}{\partial t} \\
& +2 a_{3} \frac{\partial u_{1}}{\partial t} \frac{\partial u_{2}}{\partial t}+b_{1} u_{1}^{3}+b_{2} u_{1}^{2} \frac{\partial u_{1}}{\partial t}+b_{3} u_{1}\left(\frac{\partial u_{1}}{\partial t}\right)^{2}+b_{4}\left(\frac{\partial u_{1}}{\partial t}\right)^{3}
\end{aligned}
$$

явньй вид (1.21), (1.23), (1.24) функций $u_{1}, u_{2}$ и приравнивая к нулю коэффициенты при гармониках вида (1.26), для определения постоянной $\xi$ приходим к уравнению

$$
(-\omega+i \delta) \xi+\frac{1}{2} i \alpha \bar{\xi}+d|\xi|^{2} \xi=0
$$


где

$$
\begin{aligned}
d= & \omega a_{2}\left(a_{1}+\omega^{2} a_{3}\right)\left(\frac{3}{4}+\frac{1}{2\left(4 \omega^{2}-3\right)}-\frac{3}{2\left(4 \omega^{2}-1\right)}\right) \\
& +\frac{3}{4}\left(\omega b_{2}+3 \omega^{3} b_{4}\right)-2 i a_{1}\left(a_{1}+\omega^{2} a_{3}\right)\left(1+\frac{1}{2\left(4 \omega^{2}-3\right)}\right) \\
& +i\left[\left(a_{1}-\omega^{2} a_{3}\right)\left(a_{1}+2 \omega^{2} a_{3}\right)-\frac{1}{2} a_{2}^{2} \omega^{2}\right]\left(\frac{1}{6}+\frac{1}{4 \omega^{2}-1}\right)-\frac{3}{4} i\left(3 b_{1}+\omega^{2} b_{3}\right)
\end{aligned}
$$

Полагая $z=|\xi|^{2}$ в (1.27), для определения $z$ получим уравнение

$$
P(z) \equiv(\omega-\operatorname{Re} d \cdot z)^{2}+(\delta+\operatorname{Im} d \cdot z)^{2}-\frac{1}{4} \alpha^{2}=0
$$

исследование которого проведем при дополнительных предположениях:

$$
d \neq 0, \quad \operatorname{Re} d \leqslant 0
$$

Заметим, далее, что поскольку $\delta$ в (1.14) меняется на отрезке (1.15), то, очевидно, $P(0)<0$. А отсюда и из (1.30) вытекает, что уравнение $(1.29)$ имеет единственный положительный корень $z_{0}$, причем

$$
P^{\prime}\left(z_{0}\right)>0
$$

Таким образом, сначала из (1.27) находим

$$
\xi=\sqrt{z_{0}} \exp \left(i \varphi_{0}\right), \quad \varphi_{0}=\frac{1}{2} \operatorname{arctg} \frac{\operatorname{Re} d \cdot z_{0}-\omega}{\operatorname{Im} d \cdot z_{0}+\delta}
$$

а затем определяем и функцию $u_{3}$, представляющую собой линейную комбинацию гармоник вида (1.25) при $m=1, p=3$ и $m=3, p=1,3$. И, наконец, при $k=4$ разрешимость задачи (1.22) в классе тригонометрических многочленов обеспечивает неравенство

$$
\omega \neq \frac{\sqrt{5}}{2},
$$

возникающее при нахождении коэффициентов функции $u_{4}$ при гармониках $\exp ( \pm 2 i \omega t) \cos 4 x$.

Итак, при условиях (1.30), (1.33) приближенное периодическое решение (1.20) может быть построено с точностью до $\varepsilon^{5 / 2}$ по невязке. Линеаризуя на нем уравнение (1.14), получим краевую задачу

$$
\begin{aligned}
h_{t t} & -\left(\sigma^{2}+\varepsilon \delta\right) h_{x x}+h \\
& =\sqrt{\varepsilon}\left[a_{1}(t, x) h+a_{2}(t, x) h_{t}\right]+\varepsilon\left[b_{1}(t, x, \varepsilon) h+b_{2}(t, x, \varepsilon) h_{t}\right],\left.\quad h_{x}\right|_{x=0, \pi}=0
\end{aligned}
$$


c $(2 \pi / \omega)$-периодическими по $t$ коэффициентами, в которой

$$
\begin{gathered}
a_{1}(t, x)=2 a_{1} u_{1}+a_{2} \frac{\partial u_{1}}{\partial t}, \quad a_{2}(t, x)=a_{2} u_{1}+2 a_{3} \frac{\partial u_{1}}{\partial t} \\
b_{1}(t, x, 0)=-\alpha \cos 2 \omega t+2 a_{1} u_{2}+a_{2} \frac{\partial u_{2}}{\partial t}+3 b_{1} u_{1}^{2}+2 b_{2} u_{1} \frac{\partial u_{1}}{\partial t}+b_{3}\left(\frac{\partial u_{1}}{\partial t}\right)^{2} \\
b_{2}(t, x, 0)=-1+a_{2} u_{2}+2 a_{3} \frac{\partial u_{2}}{\partial t}+b_{2} u_{1}^{2}+2 b_{3} u_{1} \frac{\partial u_{1}}{\partial t}+3 b_{4}\left(\frac{\partial u_{1}}{\partial t}\right)^{2}
\end{gathered}
$$

Как известно [15], основной этап доказательства существования у задачи (1.14) периодического решения с асимптотикой (1.20) состоит в исследовании свойств устойчивости решений уравнения (1.34) и в обратимости соответствуюшего ему дифференциального оператора (в подходящем пространстве периодических функций). Обе эти проблемы решаются с помощью алгоритма анализа устойчивости из [11], адаптированного в [14] для телеграфных уравнений. Описание указанного алгоритма приводится ниже.

Начнем с расчета характеристических показателей (т.е. логарифмов мультипликаторов, деленных на период) уравнения (1.34), отвечаюших генерируемой моде $\cos x$. Следуя методике [11], положим в (1.34)

$$
\begin{aligned}
h & =\left[V_{0}(t, x)+\sqrt{\varepsilon} V_{1}(t, x)+\varepsilon V_{2}(t, x)\right] \exp (\varepsilon D d), \\
V_{j} & =\left[w_{j}, \bar{w}_{j}\right], \quad j=0,1,2, \quad w_{0}=\exp (i \omega t) \cos x,
\end{aligned}
$$

где $w_{j}$ - тригонометрические полиномы переменной $\omega t$, а матрица $D$ имеет структуру

$$
\left(\begin{array}{ll}
d_{1} & d_{2} \\
\bar{d}_{2} & \bar{d}_{1}
\end{array}\right)
$$

Приравнивая коэффициенты при $\sqrt{\varepsilon}$ и $\varepsilon$, для определения $w_{1}$ и $w_{2}$ получим краевые задачи вида (1.22), первая из которых разрешима в нужном классе функций. Точнее говоря, формула для $w_{1}$ получается при дифференцировании равенства (1.23) по $\xi$ (переменные $\xi$ и $\bar{\xi}$ при этом считаются независимыми). Далее, неоднородность в уравнении для $w_{2}$ представляет собой линейную комбинацию гармоник (1.25). Поэтому, как и в случае задачи (1.22) при $k=3$, приравняем к нулю коэффициенты при гармониках (1.26). В результате получим уравнения для определения $d_{1}$ и $d_{2}$, из которых находим

$$
d_{1}=-\frac{1}{2}+\frac{i \delta}{2 \omega}+\frac{d}{\omega}|\xi|^{2}, \quad d_{2}=\frac{i \alpha}{4 \omega}+\frac{d}{2 \omega} \xi^{2}
$$

После этого определим функцию $w_{2}$, структура которой аналогична $u_{3}$. Отметим, наконец, что из (1.39) и неравенств $(1.30),(1.31)$ вытекает гурвицевость матрицы (1.38).

При $m=0,2,3, \ldots$ подставим в (1.34) представление

$$
h=\left[\exp \left(i \omega_{m} \tau_{m}\right) \cos m x+\sqrt{\varepsilon} h_{1 m}\left(t, \tau_{m}, x\right)+\varepsilon h_{2 m}\left(t, \tau_{m}, x\right)\right] \exp \left(\varepsilon \mu_{m} t\right),
$$


где частоты $\omega_{m}$ задаются равенствами из $(1.19) ; \tau_{m}=\left(1+\varepsilon m^{2} \delta / \omega_{m}^{2}\right)^{1 / 2} t$, функции $h_{1 m}, h_{2 m}$ - тригонометрические полиномы переменных $\omega_{m} \tau_{m}, \omega t$, a $\mu_{m}$ - подлежащая определению комплексная постоянная. В результате, приравнивая коэффициенты при $\sqrt{\varepsilon}$, для $h_{1 m}$ получим задачу

$$
\begin{gathered}
\left(\frac{\partial^{2}}{\partial t^{2}}+2 \frac{\partial^{2}}{\partial t \partial \tau_{m}}+\frac{\partial^{2}}{\partial \tau_{m}^{2}}-\sigma^{2} \frac{\partial^{2}}{\partial x^{2}}\right) h_{1 m}+h_{1 m} \\
=\left[a_{1}(t, x)+i \omega_{m} a_{2}(t, x)\right] \exp \left(i \omega_{m} \tau_{m}\right) \cos m x \\
\left.\frac{\partial h_{1 m}}{\partial x}\right|_{x=0, \pi}=0
\end{gathered}
$$

решение которой будем искать в виде тригонометрического многочлена той же структуры, что и правая часть ее уравнения. На этом пути с учетом равенств (1.35) приходим к выводу, что

$$
\begin{aligned}
h_{1 m}=\{ & \left.v_{1 m} \cos [(m+1) x]+v_{2 m} \cos [(m-1) x]\right\} \exp \left[i\left(\omega_{m} \tau_{m}+\omega t\right)\right] \\
& +\left\{v_{3 m} \cos [(m+1) x]+v_{4 m} \cos [(m-1) x]\right\} \exp \left[i\left(\omega_{m} \tau_{m}-\omega t\right)\right]
\end{aligned}
$$

где

$$
\begin{gathered}
v_{1 m}=\frac{v_{+}}{\omega_{m+1}^{2}-\left(\omega_{m}+\omega\right)^{2}}, \quad v_{2 m}=\frac{v_{+}}{\omega_{m-1}^{2}-\left(\omega_{m}+\omega\right)^{2}} \\
v_{3 m}=\frac{v_{-}}{\omega_{m+1}^{2}-\left(\omega_{m}-\omega\right)^{2}}, \quad v_{4 m}=\frac{v_{-}}{\omega_{m-1}^{2}-\left(\omega_{m}-\omega\right)^{2}} \\
v_{+}=\frac{1}{2} \xi\left[2 a_{1}+i\left(\omega_{m}+\omega\right) a_{2}-2 \omega_{m} \omega a_{3}\right] \\
v_{-}=\frac{1}{2} \bar{\xi}\left[2 a_{1}+i\left(\omega_{m}-\omega\right) a_{2}+2 \omega_{m} \omega a_{3}\right] .
\end{gathered}
$$

Отметим, что в (1.43) при любом $\sigma>0$

$$
\left(\omega+\omega_{m}\right)^{2}-\omega_{m \pm 1}^{2} \neq 0, \quad\left(\omega-\omega_{m}\right)^{2}-\omega_{m \pm 1}^{2} \neq 0 .
$$

Действительно, в справедливости первого неравенства (1.45) убеждаемся, переписывая его в эквивалентной форме $-\frac{1}{2} \pm \sigma^{2} m \neq \sqrt{1+\sigma^{2}} \sqrt{1+\sigma^{2} m^{2}}$ и замечая, что в силу неравенства Буняковского-Коши

$$
\left|-\frac{1}{2} \pm \sigma^{2} m\right| \leqslant \sqrt{\frac{1}{4}+\sigma^{2}} \sqrt{1+\sigma^{2} m^{2}}<\sqrt{1+\sigma^{2}} \sqrt{1+\sigma^{2} m^{2}}
$$

Второе неравенство (1.45) устанавливается аналогично. Отметим также, что при $m \rightarrow \infty$ коэффициенты (1.43) стремятся к конечным пределам, а значит,

$$
\left|v_{j m}\right| \leqslant N, \quad j=1, \ldots, 4
$$

здесь и ниже через $N$ обозначаем различные универсальные (не зависящие от $m, \varepsilon$ ) положительные постоянные, точные значения которых несущественны. 
При нахождении $h_{2 m}$ имеем дело с аналогичной (1.41) задачей. Учитывая (1.35), (1.36), (1.42)-(1.44) в ее правой части, имеющей вид

$$
\begin{aligned}
g= & a_{1}(t, x) h_{1 m}+a_{2}(t, x)\left(\frac{\partial h_{1 m}}{\partial t}+\frac{\partial h_{1 m}}{\partial \tau_{m}}\right) \\
& +\left(b_{1}(t, x, 0)+i \omega_{m} b_{2}(t, x, 0)\right) \exp \left(i \omega_{m} \tau_{m}\right) \cos m x
\end{aligned}
$$

и приравнивая в $g$ к нулю коэффициент при гармонике $\exp \left(i \omega_{m} \tau_{m}\right) \cos m x$, для определения $\mu_{m}$ приходим к равенству

$$
\begin{aligned}
2 i \omega_{m} \mu_{m}+i \omega_{m}= & \frac{1}{2} \bar{\xi}\left(v_{1 m}+v_{2 m}\right)\left[2 a_{1}-i \omega a_{2}+i\left(\omega_{m}+\omega\right)\left(a_{2}-2 i \omega a_{3}\right)\right] \\
& +\frac{1}{2} \xi\left(v_{3 m}+v_{4 m}\right)\left[2 a_{1}+i \omega a_{2}+i\left(\omega_{m}-\omega\right)\left(a_{2}+2 i \omega a_{3}\right)\right] \\
& +|\xi|^{2}\left[2 a_{1}\left(a_{1}+\omega^{2} a_{3}\right)+3 b_{1}+\omega^{2} b_{3}\right] \\
& +i \omega_{m}|\xi|^{2}\left[a_{2}\left(a_{1}+\omega^{2} a_{3}\right)+b_{2}+3 \omega^{2} b_{4}\right]
\end{aligned}
$$

Из (1.47), в частности, следует, что при $m \rightarrow \infty$ последовательность $\mu_{m}$ имеет конечный предел $\mu_{\infty}$.

После определения $\mu_{m}$ в $g\left(t, \tau_{m}, x\right)$ остаются лиш гармоники

$$
\begin{array}{ll}
\exp \left(i \omega_{m} \tau_{m}\right) \cos [(m \pm 2) x], & \exp \left[i\left(\omega_{m} \tau_{m}+2 \omega t\right)\right] \cos [(m \pm 2) x], \\
\exp \left[i\left(\omega_{m} \tau_{m}-2 \omega t\right)\right] \cos [(m \pm 2) x], & \exp \left[i\left(\omega_{m} \tau_{m} \pm 2 \omega t\right)\right] \cos m x
\end{array}
$$

Поэтому для нахождения $h_{2 m}$ в том же классе функций следует убедиться в справедливости неравенств

$$
\begin{array}{ll}
\omega_{m}^{2}-\omega_{m \pm 2}^{2} \neq 0, & \left(\omega_{m}+2 \omega\right)^{2}-\omega_{m \pm 2}^{2} \neq 0 \\
\left(\omega_{m}-2 \omega\right)^{2}-\omega_{m \pm 2}^{2} \neq 0, & \left(\omega_{m} \pm 2 \omega\right)^{2}-\omega_{m}^{2} \neq 0 .
\end{array}
$$

Второе и третье неравенства (1.49) эквивалентны условию

$$
\left|1 \pm \sigma^{2} m\right| \neq \sqrt{1+\sigma^{2} m^{2}} \sqrt{1+\sigma^{2}}
$$

а так как $m \neq 1$, то в силу неравенства Буняковского-Коши левая часть (1.50) строго менњше правой. Первое и четвертое неравенства (1.50) эквивалентны соответственно очевидным неравенствам $4 \sigma^{2}(m \pm 1) \neq 0$ и $4 \omega\left(\omega \pm \omega_{m}\right) \neq 0$. Отметим, что выражения в левых частях неравенств (1.49) после деления на $\omega_{m}$ имеют при $m \rightarrow \infty$ ненулевые конечные пределы; отсюда, в свою очередь, следует, что для коэффициентов функции $h_{2 m}$ при гармониках (1.48) справедливы аналогичные (1.46) оценки. 
Перед формулировкой основного результата введем в рассмотрение функции $R_{m}(\delta)=\operatorname{Re} \mu_{m}(m \neq 1), R_{\infty}(\delta)=\operatorname{Re} \mu_{\infty}$, для которых из (1.47) вытекают равенства

$$
\begin{aligned}
2 R_{m}(\delta)= & -1+\frac{1}{2} a_{2}\left(a_{1}+\omega^{2} a_{3}\right)\left(2+\frac{\omega}{\omega_{m}}\right) \\
& \times\left[\frac{1}{\omega_{m+1}^{2}-\left(\omega_{m}+\omega\right)^{2}}+\frac{1}{\omega_{m-1}^{2}-\left(\omega_{m}+\omega\right)^{2}}\right] z_{0}(\delta) \\
& +\left[a_{2}\left(a_{1}+\omega^{2} a_{3}\right)+b_{2}+3 \omega^{2} b_{4}\right] z_{0}(\delta)+\frac{1}{2} a_{2}\left(a_{1}+\omega^{2} a_{3}\right) \\
& \times\left(2-\frac{\omega}{\omega_{m}}\right)\left[\frac{1}{\omega_{m+1}^{2}-\left(\omega_{m}-\omega\right)^{2}}+\frac{1}{\omega_{m-1}^{2}-\left(\omega_{m}-\omega\right)^{2}}\right] z_{0}(\delta), \\
2 R_{\infty}(\delta)= & -1+\left[a_{2}\left(a_{1}+\omega^{2} a_{3}\right)+b_{2}+3 \omega^{2} b_{4}\right] z_{0}(\delta),
\end{aligned}
$$

где $z_{0}=z_{0}(\delta)-$ положительный корень уравнения (1.29).

Лемма 2. Пусть выполнены неравенства (1.30), (1.33). Пусть, далее, при всех $\delta$ из отрезка (1.15) отличны от нуля функиии (1.51), (1.52) и количество положстельных среди них равно $m_{0}$. Тогда найдется такое $\varepsilon_{0}>0$, что при всех $0<\varepsilon \leqslant \varepsilon_{0}, \delta_{1} \leqslant \delta \leqslant \delta_{2}$ краевая задача (1.14) имеет два периодических nо $t$ с периодом $2 \pi / \omega$ решения

$$
u_{0}^{1}(t, x, \varepsilon, \delta)=u_{0}(t, x, \varepsilon, \delta), \quad u_{0}^{2}(t, x, \varepsilon, \delta)=u_{0}(t, \pi-x, \varepsilon, \delta)
$$

где функиия $u_{0}$ допускает (в метрике $\stackrel{\circ}{\mathrm{W}} \underset{2}{2}(0, \pi)$ ) равномерное по $t$ асимптотическое представление (1.20), (1.21), (1.32). Периодические решения (1.53) экспоненииально устойчивы в случае $m_{0}=0$ и дихотомичны в случае $m_{0}>0$ с размерностью неустойчивого многообразия $2 m_{0}$.

Сформулированная лемма и описанный в предыдущем пункте принцип подобия приводят к следующему утверждению.

ТЕОРЕМА 1. При выполнении условий леммы 2 исходная краевая задача (1.1) при всех $0<\varepsilon \ll 1$ имеет периодические решения (1.17), (1.18), причем в качестве функиии (1.16) фигурирует периодическое решение $u_{0}(t, x, \varepsilon, \delta)$ из (1.53).

Отметим, что количество $n_{* *}(\varepsilon)$ номеров $n$, удовлетворяющих оценкам (1.18), в принципе близко к числу $n_{*}(\varepsilon)$ возбужденных мод. Точнее говоря, для $n_{* *}(\varepsilon)$ справедлива аналогичная (1.12) формула, в которой $\delta_{*}$ следует заменить на $\left(\delta_{2}-\delta_{1}\right) / 2$. А отсюда, в свою очередь, заключаем, что количество периодических решений краевой задачи (1.1), доставляемых теоремой 1 , неограниченно увеличивается как при увеличении $\alpha$ (в этом случае одновременно с $\alpha$ надо увеличивать и $\delta_{2}-\delta_{1}$ ), так и при $\omega \rightarrow 1, \varepsilon \rightarrow 0$. Отметим еще, что последний способ увеличения $n_{* *}(\varepsilon)$ менее ограничителен, так как в этом случае в качестве $(1.15)$ можно брать любой фиксированный отрезок $\left[\delta_{1}, \delta_{2}\right] \subset\left(-\sqrt{\alpha^{2} / 4-1}, \sqrt{\alpha^{2} / 4-1}\right)$. 
1.4. Доказательство леммы 2. Для начала обоснуем описанньй выше алгоритм асимптотического вычисления характеристических показателей задачи (1.34). Введем новые переменные $h_{1}=h_{t}, h_{2}=B h$, где $B$ - арифметический квадратный корень из оператора $-\left(\sigma^{2}+\varepsilon \delta\right) d^{2} / d x^{2}+I$ с граничньми условиями Неймана, и перейдем обычным образом от уравнения (1.34) к системе первого порядка в $W_{2}^{1} \times W_{2}^{1}$. Выполним затем в получившейся системе замену, исходя из формулы

$$
\begin{aligned}
h=( & \left.V_{0}+\sqrt{\varepsilon} V_{1}+\varepsilon V_{2}\right) v \\
& +\sum_{m=0,2,3, \ldots}\left\{\omega_{m}^{-2}\left[\exp \left(i \omega_{m} \tau_{m}\right) \cos m x+\sqrt{\varepsilon} h_{1 m}+\varepsilon h_{2 m}\right] \eta_{m}\right. \\
& \left.+\omega_{m}^{-2}\left[\exp \left(-i \omega_{m} \tau_{m}\right) \cos m x+\sqrt{\varepsilon} \bar{h}_{1 m}+\varepsilon \bar{h}_{2 m}\right] \bar{\eta}_{m}\right\}
\end{aligned}
$$

где $v=\operatorname{colon}\left(\eta_{1}, \bar{\eta}_{1}\right), \eta=\operatorname{colon}\left(\eta_{0}, \bar{\eta}_{0}, \eta_{1}, \bar{\eta}_{1}, \eta_{2}, \bar{\eta}_{2}, \ldots\right) \in l_{2}$. Последнее означает, что при дифференцировании (1.54) по $t$ следует считать:

$$
\dot{v}=\varepsilon D v, \quad \dot{\eta}_{m}=\varepsilon \mu_{m} \eta_{m}, \quad m \neq 1
$$

Заметим, далее, что из способа построения и свойств входящих в (1.54) функций следует, что эта формула индуцирует ограниченный равномерно по $t, \varepsilon$ (вместе со своим обратным) оператор, действующий из $l_{2}$ в $W_{2}^{1} \times W_{2}^{1}$, а результатом проведения указанной замены в (1.34) служит система в $l_{2}$ :

$$
\dot{\eta}=\varepsilon \Lambda_{0} \eta+\varepsilon^{3 / 2} \Lambda_{1}(t, \varepsilon) \eta
$$

Здесь $\Lambda_{0}=\operatorname{diag}\left\{\mu_{0}, \bar{\mu}_{0}, D, \mu_{1}, \bar{\mu}_{1}, \ldots, \mu_{m}, \bar{\mu}_{m}, \ldots\right\}$, а линейный оператор $\Lambda_{1}$ ограничен равномерно по $t \in \mathbb{R}$ и $\varepsilon$ (по поводу построения $\Lambda_{1}$ см. [7], [14]).

Несложный анализ системы (1.55) показывает, что свойства ее устойчивости совпадают с теми, о которых говорится в лемме 2: реальные части всех ее характеристических показателей равномерно по $m \geqslant 0$ с точностью до $\varepsilon^{3 / 2}$ совпадают с числами $\varepsilon \operatorname{Re} \mu_{m}, m \neq 1$, и с реальньми частями собственных значений матрицы $\varepsilon D$, которая является гурвицевой. Далее, пусть $C\left(W_{2}^{1} \times W_{2}^{1}\right)$ - банахово пространство непрерывных вектор-функций $h(t, x)$ со значениями в $W_{2}^{1} \times W_{2}^{1}$ и нормой

$$
\|h\|_{C\left(W_{2}^{1} \times W_{2}^{1}\right)}=\sup _{t}\|h(t, x)\|_{W_{2}^{1} \times W_{2}^{1}}
$$

Введем оператор П, порожденный в пространстве $C\left(W_{2}^{1} \times W_{2}^{1}\right)$ уравнением (1.34) (после перехода к переменным $\left(h_{t}, B h\right)$ ). Структура уравнения (1.55) позволяет заключить, что оператор П обратим и

$$
\left\|\Pi^{-1}\right\|_{C\left(W_{2}^{1} \times W_{2}^{1}\right) \rightarrow C\left(W_{2}^{1} \times W_{2}^{1}\right)} \leqslant \frac{N}{\varepsilon} .
$$

Завершающий этап - доказательство существования требуемого периодического решения - стандартен. Положим в (1.14)

$$
u=\sqrt{\varepsilon} u_{1}(t, x)+\varepsilon u_{2}(t, x)+\varepsilon^{3 / 2} u_{3}(t, x)+\varepsilon^{2} u_{4}(t, x)+\varepsilon^{3 / 2} h,
$$


где функции $u_{j}, j=1, \ldots, 4$, построены в предыдущем пункте. Затем перейдем к системе для $\left(u_{t}, B u\right)$, а после этого, выделяя линейную по $h$ часть и обращая оператор П, получим интегральное уравнение в $C\left(W_{2}^{1} \times W_{2}^{1}\right)$. Наконец, применяя к построенному интегральному уравнению в некотором шаре не зависящего от $\varepsilon$ радиуса с центром в нуле упомянутого пространства принцип сжимающих отображений (справедливость которого гарантирует оценка (1.56)), определим фигурирующую в (1.57) $(2 \pi / \omega)$-периодическую по $t$ функцию $h(t, x, \varepsilon, \delta)$ :

$$
\max _{t}\left(\left\|\frac{\partial h}{\partial t}\right\|_{W_{2}^{1}}+\|B h\|_{W_{2}^{1}}\right) \leqslant N
$$

Лемма 2 доказана.

\section{§2. Исследование устойчивости высокомодовых параметрических колебаний}

2.1. Описание специальных алгоритмов. Линеаризуем краевую задачу (1.1) на произвольном решении $u_{n}^{1}$ семейства $(1.17),(1.18)$ с номером $n$ (случай периодического решения $u_{n}^{2}$ не нуждается в отдельном рассмотрении, так как сводится к данному заменой $\pi-n x \rightarrow n x)$. Выполняя, далее, замену $n x \rightarrow x$, приходим к краевой задаче

$$
\begin{gathered}
h_{t t}-\left(\sigma^{2}+\varepsilon \delta\right) h_{x x}+h=\sqrt{\varepsilon}\left[a_{1}(t, x) h+a_{2}(t, x) h_{t}\right]+\varepsilon\left[b_{1}(t, x, \varepsilon) h+b_{2}(t, x, \varepsilon) h_{t}\right], \\
\left.h_{x}\right|_{x=0, n \pi}=0
\end{gathered}
$$

в которой параметр $\delta$, имеющий порядок единицы и принимающий дискретные значения (1.5), для удобства будем считать непрерьвно меняющимся на отрезке (1.15). Происхождение коэффициентов уравнения (2.1) очевидно, так как оно представляет собой линеаризацию уравнения (1.14) на периодическом решении (1.16). Однако, в отличие от задачи (1.34) задача (2.1) сингулярна, ибо рассматривается на асимптотически большом (порядка $\varepsilon^{-1}$ ) отрезке $0 \leqslant x \leqslant n \pi$.

Итак, проблема устойчивости периодических решений (1.17) сводится к анализу расположения характеристических показателей задачи (2.1). Для их расчета снова воспользуемся алгоритмом из п. 1.3. С этой целью положим в (2.1)

$$
\begin{aligned}
& h=\left[\exp \left(i \omega_{z} \tau_{z}\right) \cos z x+\sqrt{\varepsilon} h_{1 z}\left(t, \tau_{z}, x\right)+\varepsilon h_{2 z}\left(t, \tau_{z}, x\right)\right] \exp \left(\varepsilon \mu_{z} t\right), \\
& \omega_{z}=\sqrt{1+\sigma^{2} z^{2}}, \quad \tau_{z}=\left(1+\varepsilon \delta \frac{z^{2}}{\omega_{z}^{2}}\right)^{1 / 2} t, \quad z=\frac{k}{n}, \quad k=0,1, \ldots,
\end{aligned}
$$

где $h_{1 z}, h_{2 z}$ - тригонометрические многочлены переменных $\omega_{z} \tau_{z}$ и $\omega t$, a $\mu_{z}-$ подлежашая определению комплексная постоянная.

Сравнивая уравнения (1.34) и (2.1), формулы (1.40) и (2.2), убеждаемся, что $h_{1 z}, h_{2 z}, \mu_{z}$ получаются из построенных в п. 1.3 функций $h_{1 m}, h_{2 m}$ и постоянной $\mu_{m}$ посредством замены $m$ на $z$. Более того, для входящих в $h_{1 z}$ коэффициентов $v_{j z}$, 
$j=1, \ldots, 4$, оценки (1.46) сохраняются равномерно по всем $z \geqslant 0$. В случае же $h_{2 z}$ ситуация несколько иная. Здесь коэффициенты при гармониках

$$
\begin{array}{ll}
\exp \left(i \omega_{z} \tau_{z}\right) \cos [(2+z) x], & \exp \left[i\left(\omega_{z} \tau_{z}+2 \omega t\right)\right] \cos [(2 \pm z) x] \\
\exp \left[i\left(\omega_{z} \tau_{z}-2 \omega t\right)\right] \cos [(2+z) x], & \exp \left[i\left(\omega_{z} \tau_{z}+2 \omega t\right)\right] \cos z x
\end{array}
$$

допускают равномерные по всем $z \geqslant 0$ оценки вида (1.46); коэффицциенты при гармониках $\exp \left(i \omega_{z} \tau_{z}\right) \cos [(2-z) x], \exp \left[i\left(\omega_{z} \tau_{z}-2 \omega t\right)\right] \cos z x$ при $z \rightarrow 1$ имеют особенность вида $1 /(z-1)$, а коэффициент при гармонике $\exp \left[i\left(\omega_{z} \tau_{z}-2 \omega t\right)\right] \times$ $\cos [(2-z) x]$ при $z \rightarrow 1$ растет как $1 /(z-1)^{2}$. Если же параметр $z$ меняется на полуоси $z \geqslant 0$ с выброшенной фиксированной окрестностью точки $z=1$, то равномерно ограниченными по $z$ будут все входящие в $h_{2 z}$ коэффициенты.

Перед формулировкой первого ограничения, предполагая, что $z$ непрерывно меняется на полуоси $z \geqslant 0$, введем функции

$$
\varphi(z, \delta)=\operatorname{Re} \mu_{z}, \quad \varphi_{\infty}(\delta)=\operatorname{Re} \mu_{\infty} .
$$

Заметим, что $\varphi(z, \delta) \rightarrow \varphi_{\infty}(\delta)$ при $z \rightarrow \infty$ равномерно по $\delta$ из отрезка (1.15).

УСловие 1. Сиитаем, ито $\varphi(z, \delta)<0, \varphi_{\infty}(\delta)<0$ при всех $\delta_{1} \leqslant \delta \leqslant \delta_{2} u$ $z \geqslant 0$.

Сформулированное ограничение очевидньм образом усиливает предположения леммы 2 , так как $R_{m}(\delta)=\varphi(m, \delta)$.

При $z \rightarrow 1$ сталкиваемся с особенностью, вызванной так называемыми пространственно-временньми резонансами. Действительно, частота $\omega$, отвечающая генерируемой моде $\cos x$, и частоты $\omega_{1 \pm k / n}, k=1,2, \ldots$, на соседних модах $\cos [(1 \pm k / n) x]$ в силу малости $k / n$ близки. Поэтому расчет характеристических показателей задачи (2.1) на этих модах в одночастотной форме (2.2) заведомо невозможен. Для того чтобы исправить положение, при

$$
z=1 \pm \nu, \quad 0<\nu \leqslant \nu_{0}
$$

где $\nu_{0}>0$ фиксировано и достаточно мало, воспользуемся следующим специальным приемом. Положим в (2.1)

$$
h=v_{1} \cos \nu x-v_{2} \sin \nu x,
$$

где функции $v_{j}(t, x, \nu, \varepsilon), j=1,2$, таковы, что

$$
\left.\frac{\partial v_{1}}{\partial x}\right|_{x=0, \pi}=0,\left.\quad v_{2}\right|_{x=0, \pi}=0
$$

В результате для $v=\operatorname{colon}\left(v_{1}, v_{2}\right)$ получим уравнение

$$
\begin{aligned}
v_{t t}+v= & \left(\sigma^{2}+\varepsilon \delta\right)\left[v_{x x}+2 \nu A_{0} v_{x}-\nu^{2} v\right] \\
& +\sqrt{\varepsilon}\left[a_{1}(t, x) v+a_{2}(t, x) v_{t}\right]+\varepsilon\left[b_{1}(t, x, \varepsilon) v+b_{2}(t, x, \varepsilon) v_{t}\right]
\end{aligned}
$$


где

$$
A_{0}=\left(\begin{array}{cc}
0 & -1 \\
1 & 0
\end{array}\right),
$$

а параметр $\nu=k / n-1$ в силу произвольности $k$ и $n$ будем считать непрерывно меняющимся на множестве из (2.3).

Отметим, что краевая задача (2.6), (2.5) уже не содержит сингулярности в граничных условиях (вместо этого появляется дополнительный малый параметр $\nu$ ). Отметим также, что, подставляя компоненты любого ее решения в (2.4) и полагая $\nu=k / n-1$, получим, очевидно, решение исходной задачи (2.1). Таким образом, проблема расчета характеристических показателей последней на модах

$$
\cos \left(\frac{k x}{n}\right), \quad 0<\left|\frac{k}{n}-1\right| \leqslant \nu_{0},
$$

сводится к применению алгоритма из [11] к вспомогательной краевой задаче $(2.6),(2.5)$.

При $\varepsilon=0$ решениями Ляпунова-Флоке задачи $(2.6),(2.5)$ являются функции

$$
\begin{array}{ll}
\exp \left( \pm i \omega_{k+\nu} t\right) \operatorname{colon}(\cos k x, \sin k x), & k=0,1, \ldots, \\
\exp \left( \pm i \omega_{k-\nu} t\right) \operatorname{colon}(\cos k x,-\sin k x), & k=1,2, \ldots
\end{array}
$$

Однако в исходной задаче (2.1) нас интересуют моды (2.7). Поэтому ниже при реализации алгоритма из [11] будут задействованы лиш четыре функции (2.8), отвечающие номеру $k=1$.

Следуя [11], положим в (2.6), (2.5)

$$
v=\left[H_{0}+\sqrt{\varepsilon} H_{1}+\varepsilon H_{2}\right] \exp \left[\Lambda_{0}(\nu)+\varepsilon \Lambda_{1}(\nu)\right] t,
$$

где $(2 \times 4)$-матрицы $H_{k}(t, x, \nu), k=0,1,2$, и квадратные матрицы $\Lambda_{0}, \Lambda_{1}$ порядка 4 имеют вид

$$
\begin{gathered}
H_{j}=\left[w_{j 1}, \bar{w}_{j 1}, w_{j 2}, \bar{w}_{j 2}\right], \quad j=0,1,2, \quad \Lambda_{1}=\left\|\lambda_{j s}(\nu)\right\|, \quad j, s=1, \ldots, 4 \\
\lambda_{22}(\nu)=\bar{\lambda}_{11}(\nu), \quad \lambda_{21}(\nu)=\bar{\lambda}_{12}(\nu), \quad \lambda_{24}(\nu)=\bar{\lambda}_{13}(\nu), \quad \lambda_{23}(\nu)=\bar{\lambda}_{14}(\nu) \\
\lambda_{42}(\nu)=\bar{\lambda}_{31}(\nu), \quad \lambda_{41}(\nu)=\bar{\lambda}_{32}(\nu), \quad \lambda_{44}(\nu)=\bar{\lambda}_{33}(\nu), \quad \lambda_{43}(\nu)=\bar{\lambda}_{34}(\nu) \\
\Lambda_{0}=\operatorname{diag}\left\{i \sigma_{+},-i \sigma_{+}, i \sigma_{-},-i \sigma_{-}\right\}, \quad \sigma_{ \pm}(\nu)=\omega_{1 \pm \nu}-\omega, \\
w_{01}=\exp (i \omega t) \operatorname{colon}(\cos x, \sin x), \quad w_{02}=\exp (i \omega t) \operatorname{colon}(\cos x,-\sin x)
\end{gathered}
$$

Считаем еще, что столбцы матриц $H_{1}, H_{2}$ - тригонометрические полиномы переменной $\omega t$.

Приравнивая в $(2.6)$ коэффициенты при $\sqrt{\varepsilon}$, приходим к уравнениям

$$
\begin{aligned}
& \frac{\partial^{2} w_{11}}{\partial t^{2}}+2 i \sigma_{+} \frac{\partial w_{11}}{\partial t}-\sigma_{+}^{2} w_{11}-\sigma^{2}\left(\frac{\partial^{2} w_{11}}{\partial x^{2}}+2 \nu A_{0} \frac{\partial w_{11}}{\partial x}-\nu^{2} w_{11}\right)+w_{11} \\
& =a_{1}(t, x) w_{01}+a_{2}(t, x) i\left(\omega+\sigma_{+}\right) w_{01}, \\
& \frac{\partial^{2} w_{12}}{\partial t^{2}}+2 i \sigma_{-} \frac{\partial w_{12}}{\partial t}-\sigma_{-}^{2} w_{12}-\sigma^{2}\left(\frac{\partial^{2} w_{12}}{\partial x^{2}}+2 \nu A_{0} \frac{\partial w_{12}}{\partial x}-\nu^{2} w_{12}\right)+w_{12} \\
& =a_{1}(t, x) w_{02}+a_{2}(t, x) i\left(\omega+\sigma_{-}\right) w_{02},
\end{aligned}
$$


которые следует дополнить граничньми условиями типа (2.5). Решая получившшеся при этом краевые задачи, находим

$$
\begin{gathered}
w_{12}(t, x, \nu)=w_{11}(t,-x,-\nu), \\
w_{11}(t, x, \nu)=G_{-}(x, \nu)+G_{+}(x, \nu) \exp (2 i \omega t), \\
G_{ \pm}=\varkappa_{ \pm}\left[\frac{\operatorname{colon}(1,0)}{\omega_{\nu}^{2}-\left(\omega_{1+\nu} \pm \omega\right)^{2}}+\frac{\operatorname{colon}(\cos 2 x, \sin 2 x)}{\omega_{2+\nu}^{2}-\left(\omega_{1+\nu} \pm \omega\right)^{2}}\right], \\
\varkappa_{-}=\frac{1}{2} \bar{\xi}\left[2 a_{1}+i\left(\omega_{1+\nu}-\omega\right) a_{2}+2 \omega \omega_{1+\nu} a_{3}\right], \\
\varkappa_{+}=\frac{1}{2} \xi\left[2 a_{1}+i\left(\omega_{1+\nu}+\omega\right) a_{2}-2 \omega \omega_{1+\nu} a_{3}\right],
\end{gathered}
$$

где $a_{j}, j=1,2,3,-$ коэффиициенты тейлоровского разложения $(1.3), \xi$-постоянная, определяемая равенствами (1.32).

Приравнивая в (2.6) коэффициенты при $\varepsilon$, для нахождения матриц $\Lambda_{1}, H_{2}$ получаем уравнение

$$
\frac{\partial^{2} H_{2}}{\partial t^{2}}+2 \frac{\partial H_{2}}{\partial t} \Lambda_{0}+H_{2} \Lambda_{0}^{2}+H_{2}-\sigma^{2}\left[\frac{\partial^{2} H_{2}}{\partial x^{2}}+2 \nu A_{0} \frac{\partial H_{2}}{\partial x}-\nu^{2} H_{2}\right]=F
$$

в котором

$$
\begin{aligned}
F= & -2 \frac{\partial H_{0}}{\partial t} \Lambda_{1}-H_{0}\left(\Lambda_{0} \Lambda_{1}+\Lambda_{1} \Lambda_{0}\right)+a_{1}(t, x) H_{1} \\
& +a_{2}(t, x)\left(\frac{\partial H_{1}}{\partial t}+H_{1} \Lambda_{0}\right)+b_{1}(t, x, 0) H_{0} \\
& +b_{2}(t, x, 0)\left(\frac{\partial H_{0}}{\partial t}+H_{0} \Lambda_{0}\right)+\delta\left[\frac{\partial^{2} H_{0}}{\partial x^{2}}+2 \nu A_{0} \frac{\partial H_{0}}{\partial x}-\nu^{2} H_{0}\right] .
\end{aligned}
$$

Затем за счет выбора восьми неизвестных комплексных элементов матрицы $\Lambda_{1}$ (см. (2.10)) добиваемся отсутствия в столбцах матрицы (2.15) слагаемых, пропорциональных $\exp ( \pm i \omega t) \operatorname{colon}(\cos x, \sin x), \exp ( \pm i \omega t) \operatorname{colon}(\cos x,-\sin x)$. После этого решение уравнения (2.14) однозначно находится в том же виде, что и неоднородность $(2.15)$, причем в отличие от $h_{2 z}$ особенностипри $\nu \rightarrow 0$ здесь отсутствуют ("опасные" слагаемые в $F$ уничтожены за счет специального выбора $\left.\lambda_{j s}(\nu)\right)$.

В дальнейшем при $\nu \neq 0$ нам потребуется явный вид только диагональных элементов матрицы $\Lambda_{1}(\nu)$, а все остальные ее элементы достаточно знать лишш при $\nu=0$. Заметим также, что уравнение (2.6) не меняется при заменах $x \rightarrow-x$, $\nu \rightarrow-\nu$, а значит,

$$
\begin{array}{ll}
\lambda_{11}(-\nu)=\lambda_{33}(\nu), & \lambda_{21}(-\nu)=\lambda_{43}(\nu), \quad \lambda_{31}(-\nu)=\lambda_{13}(\nu), \quad \lambda_{41}(-\nu)=\lambda_{23}(\nu), \\
\lambda_{12}(-\nu)=\lambda_{34}(\nu), & \lambda_{32}(-\nu)=\lambda_{14}(\nu), \quad \lambda_{42}(-\nu)=\lambda_{24}(\nu) .
\end{array}
$$


Учитывая в $(2.15)$ соотношения $(1.35),(1.36),(2.10)-(2.13),(2.16)$ и проводя соответствующие вычисления, убеждаемся, что

$$
\begin{gathered}
\lambda_{11}(\nu)=\mu_{1+\nu}+i \delta(1+\nu)^{2}\left(2 \omega_{1+\nu}\right)^{-1}, \lambda_{22}(\nu)=\bar{\lambda}_{11}(\nu) \\
\lambda_{33}(\nu)=\lambda_{11}(-\nu), \quad \lambda_{44}(\nu)=\bar{\lambda}_{11}(-\nu) \\
\Lambda_{1}(0)=\left(\begin{array}{cc}
B_{0} & C_{0} \\
C_{0} & B_{0}
\end{array}\right), \quad B_{0}=\left(\begin{array}{ll}
\varkappa_{1} & \varkappa_{2} \\
\varkappa_{2} & \varkappa_{1}
\end{array}\right), \quad C_{0}=\left(\begin{array}{ll}
\varkappa_{3} & \varkappa_{4} \\
\varkappa_{4} & \bar{\varkappa}_{3}
\end{array}\right) \\
\varkappa_{1}=-\frac{1}{2}+\frac{i \delta}{2 \omega}+\frac{|\xi|^{2}}{2 \omega} r_{1}, \varkappa_{2}=\frac{i \alpha}{4 \omega}+\frac{\xi^{2}}{2 \omega} r_{2}, \varkappa_{3}=\frac{|\xi|^{2}}{2 \omega} r_{3}, \varkappa_{4}=\frac{\xi^{2}}{2 \omega} r_{4}, \\
r_{1}=\omega a_{2}\left(a_{1}+\omega^{2} a_{3}\right)\left(1+\frac{1}{2\left(4 \omega^{2}-3\right)}-\frac{3}{2\left(4 \omega^{2}-1\right)}\right) \\
+\omega b_{2}+3 \omega^{3} b_{3}-2 i a_{1}\left(a_{1}+\omega^{2} a_{3}\right)\left(\frac{3}{2}+\frac{1}{2\left(4 \omega^{2}-3\right)}\right) \\
+i\left[\left(a_{1}-\omega^{2} a_{3}\right)\left(a_{1}+2 \omega^{2} a_{3}\right)-\frac{1}{2} a_{2}^{2} \omega^{2}\right]\left(\frac{1}{3}+\frac{1}{4 \omega^{2}-1}\right)-i\left(3 b_{1}+\omega^{2} b_{3}\right) \\
r_{2}=r_{3}=\omega a_{2}\left(a_{1}+\omega^{2} a_{3}\right)\left(\frac{1}{2}+\frac{1}{2\left(4 \omega^{2}-3\right)}-\frac{3}{2\left(4 \omega^{2}-1\right)}\right) \\
+\frac{1}{2}\left(\omega b_{2}+3 \omega^{3} b_{3}\right)-i a_{1}\left(a_{1}+\omega^{2} a_{3}\right)\left(1+\frac{1}{4 \omega^{2}-3}\right) \\
+i\left[\left(a_{1}-\omega^{2} a_{3}\right)\left(a_{1}+2 \omega^{2} a_{3}\right)-\frac{1}{2} a_{2}^{2} \omega^{2}\right] \frac{1}{4 \omega^{2}-1}-\frac{1}{2} i\left(3 b_{1}+\omega^{2} b_{3}\right) \\
r_{4}=\frac{1}{4} \omega a_{2}\left(a_{1}+\omega^{2} a_{3}\right)+\frac{1}{4}\left(\omega b_{2}+3 \omega^{3} b_{4}\right)-i a_{1}\left(a_{1}+\omega^{2} a_{3}\right) \\
+\frac{1}{6} i\left[\left(a_{1}-\omega^{2} a_{3}\right)\left(a_{1}+2 \omega^{2} a_{3}\right)-\frac{1}{2} a_{2}^{2} \omega^{2}\right]-\frac{1}{4} i\left(3 b_{1}+\omega^{2} b_{3}\right)
\end{gathered}
$$

Подставляя компоненты $v_{1}, v_{2}$ столбцов матрицы (2.9) в (2.4) и полагая затем $\nu=k / n, k=1, \ldots, k_{0}$, где $k_{0}$ - целая часть $n \nu_{0}$, приходим к искомым формулам для приближенных решений Ляпунова-Флоке задачи (2.1) на модах (2.7). На самой же генерируемой моде $\cos x$ остаются в силе формулы (1.37)-(1.39).

УСловие 2. Предполагаем, что при всех $\delta_{1} \leqslant \delta \leqslant \delta_{2}$ us $s \geqslant 0$

$$
Q(s, \delta) \equiv\left|\varkappa_{1}\right|^{2}-\left|\varkappa_{4}\right|^{2}+s \operatorname{Re}\left(i \bar{\varkappa}_{1}\right)+\frac{1}{4} s^{2}>0 .
$$

Введем, далее, последовательность матриц

$$
\Lambda_{1}(0)+k a \sigma \omega^{-1} \operatorname{diag}\{i,-i,-i, i\}, \quad k=1,2, \ldots
$$

где $\sigma=\sqrt{\omega^{2}-1}$, а параметр $a>0$ - фигурирующий в (1.1) коэффициент перед второй производной по $x$. 
УСЛОВИЕ 3. Сиитаем, что при $\delta_{1} \leqslant \delta \leqslant \delta_{2}$ все матрицы (2.24) являются гурвицевыми.

Теперь можно дать ответ на вопрос об устойчивости периодических решений (1.17), (1.18). Справедливо следуюшее утверждение.

ТЕОРЕМА 2. Пусть в дополнение к неравенствам (1.30), (1.33) выполнень условия 1-3. Тогда при всех достаточно малых $\varepsilon>0$ периодические решения (1.17), (1.18) краевой задачи (1.1) әкспоненциально устойчивы.

2.2. Доказательство теоремы 2. Используя построенные по описанным выше алгоритмам функции, сконструируем аналогичную (1.54) замену переменных, действуюшую из $l_{2}$ в $W_{2}^{1}(0, n \pi) \times W_{2}^{1}(0, n \pi)$ и преобразующую исходную задачу (2.1) в уравнение вида (1.55):

$$
\dot{\eta}=\varepsilon \Gamma_{0} \eta+\varepsilon^{3 / 2} \Gamma_{1}(t, \varepsilon) \eta
$$

Здесь $\eta \in l_{2}$, линейный оператор $\Gamma_{1}$ ограничен равномерно по всем $t \in \mathbb{R}$ и $\varepsilon$,

$$
\begin{gathered}
\Gamma_{0}=\operatorname{diag}\left\{\gamma_{0}, \bar{\gamma}_{0}, \ldots, \gamma_{n-k_{0}-1}, \bar{\gamma}_{n-k_{0}-1}, D, \Lambda_{1}, \ldots, \Lambda_{k_{0}}\right. \\
\left.\gamma_{n+k_{0}+1}, \bar{\gamma}_{n+k_{0}+1}, \ldots, \gamma_{m}, \bar{\gamma}_{m}, \ldots\right\}
\end{gathered}
$$

где $D$ - матрица (1.38),

$$
\begin{array}{ll}
\gamma_{m}=\left.\mu_{z}\right|_{z=m / n}, & m=0,1, \ldots, \\
\Lambda_{k}=\left.\varepsilon^{-1}\left[\Lambda_{0}(\nu)+\varepsilon \Lambda_{1}(\nu)\right]\right|_{\nu=k / n}, & k=1, \ldots, k_{0},
\end{array}
$$

а $k_{0}$ - целая часть $n \nu_{0}($ см. $(2.7))$.

Свойства устойчивости уравнения (2.25) очевидны и определяются по его главной части, т.е. по расположению спектра оператора $\Gamma_{0}$. Спектр же последнего это последовательность собственных чисел

$$
\gamma_{m}, \bar{\gamma}_{m}: m \geqslant 0, \quad m \neq n \pm k, \quad k=0,1, \ldots, k_{0}, \quad \gamma_{\infty}=\lim \gamma_{m}, \quad m \rightarrow \infty
$$

имеющих в силу (2.27) и условия 1 отрицательные действительные части, и собственные значения матриц $D, \Lambda_{k}, k=1, \ldots, k_{0}$, первая из которых, напомним, гурвицева.

Итак, за устойчивость периодических решений $(1.17),(1.18)$ отвечают в конечном итоге четырехмерные матрицы $\Lambda_{k}, k=1, \ldots, k_{0}$. При $k / n \sim 1$ их гурвицевость снова вытекает из условия 1. Действительно, в этом случае поправками порядка $\varepsilon$ к чисто мнимым собственным значениям матрицы $\Lambda_{0}(\nu)$ являются диагональные элементы матрицы $\varepsilon \Lambda_{1}(\nu)$ (см. (2.17)), имеющие отрицательные действительные части.

При $\nu \sim \sqrt{\varepsilon}$ разность между собственными значениями $i \sigma_{+}$и $-i \sigma_{-}$матрицы $\Lambda_{0}(\nu)$ становится величиной порядка $\varepsilon$. Полагая в этом случае $\nu^{2}=s \sigma^{2} \varepsilon / \omega^{3}$, 
$s \sim 1$, приходим к выводу, что поправками порядка $\varepsilon$ к чисто мнимьм собственным числам $\Lambda_{0}(\nu)$ теперь являются собственные значения матриц $\varepsilon \Omega(s), \varepsilon \bar{\Omega}(s)$, где

$$
\Omega(s)=\left(\begin{array}{cc}
\varkappa_{1}+\frac{1}{2} i s & \varkappa_{4} \\
\varkappa_{4} & \varkappa_{1}-\frac{1}{2} i s
\end{array}\right), \quad s \geqslant 0 .
$$

Заметим, далее, что матрицы (2.28) гурвицевы при всех $s \geqslant 0$, так как в силу условий 1 и 2 имеем: $\operatorname{sp} \Omega(s)=2 \operatorname{Re} \varkappa_{1}<0, \operatorname{det} \Omega(s)=Q(s, \delta)>0$.

И наконец, при $k \sim 1$ подставим в $\Lambda_{k}$ равенство $\nu=a \varepsilon k / \sigma$ и переразложим результат по $\varepsilon$. В итоге убеждаемся, что пределами матриц $\Lambda_{k}$ при $\varepsilon \rightarrow 0$ здесь являются матрицы (2.24), гурвицевость которых предполагается в условии 3 . Остается заметить, что тремя рассмотренными случаями исчерпываются все принципиально различные ситуации. Действительно, при $s \rightarrow \infty$ действительные части собственных чисел матриц (2.28) стремятся к Re $\varkappa_{1}$, т.е. осуществляется непрерывный переход между случаями $\nu \sim 1$ и $\nu \sim \sqrt{\varepsilon}$. Далее, при $k \rightarrow \infty$ действительные части собственных чисел матриц (2.24) стремятся к действительным частям собственных значений матрицы (2.28) при $s=0$, т.е. имеется стыковка и между случаями $\nu \sim \sqrt{\varepsilon}$ и $\nu \sim \varepsilon$. Таким образом, гурвицевьми являются все матрицы $\Lambda_{k}$, $k=1, \ldots, k_{0}$. Теорема 2 доказана.

2.3. Заключительные замечания. Реализуемость условий $1-3$ проиллюстрируем на двух примерах. В качестве первого возьмем краевую задачу

$$
u_{t t}+\varepsilon u_{t}+(1+\varepsilon \alpha \cos 2 \omega t) u=\varepsilon^{2} a^{2} u_{x x}-u^{2} u_{t},\left.\quad u_{x}\right|_{x=0}=\left.u_{x}\right|_{x=\pi}=0 .
$$

Отметим сразу, что в данном случае ограничения (1.30) выполняются, так как $d=-3 \omega / 4$. Кроме того, в (1.20) имеем $u_{2}=u_{4}=0$ и, следовательно, отпадает надобность в неравенстве (1.33). Не вызывает затруднений и проверка условий 1 и 2 , так как здесь

$$
2 \varphi(z, \delta)=-1-z_{0}<0, \quad Q(s, \delta)=\frac{1}{4}\left(1+\frac{3}{4} z_{0}\right)\left(1+\frac{5}{4} z_{0}\right)+\frac{1}{4}\left(s+\frac{\delta}{\omega}\right)^{2}>0
$$

где $z_{0}$ - положительный корень уравнения (1.29).

Итак, в проверке нуждается только гурвицевость матриц (2.24), в которых (см. $(2.18)-(2.22))$

$$
\varkappa_{1}=-\frac{1}{2}+\frac{i \delta}{2 \omega}-\frac{1}{2}|\xi|^{2}, \quad \varkappa_{2}=\frac{i \alpha}{4 \omega}-\frac{\xi^{2}}{4}, \quad \varkappa_{3}=-\frac{1}{4}|\xi|^{2}, \quad \varkappa_{4}=-\frac{1}{8} \xi^{2} .
$$

Соответствующий анализ проведем в наиболее интересном случае, когда $\alpha \gg 1$, а значит, и $|\xi|^{2} \gg 1$. С этой целью введем матрицу

$$
G(\alpha, \delta)+z \operatorname{diag}\{i,-i,-i, i\}
$$

где $G=\Lambda_{1}(0) /|\xi|^{2}$, а вспомогательньй параметр $z=k a \sigma\left(\omega|\xi|^{2}\right)^{-1}$, принимающий в силу произвольности $k$ и $|\xi|^{2} \gg 1$ фактически любое положительное значение, для удобства будем считать непрерывно меняющимся на полуоси $z>0$. 
ЛЕмма 3. По любим $\delta_{1}<\delta_{2}$ можкно указать такое достаточно большое $\alpha_{0}=\alpha_{0}\left(\delta_{1}, \delta_{2}\right)>0$, что при всех $\alpha \geqslant \alpha_{0}, z>0$ матрица (2.31) является гурвицевой.

ДоКАЗАТЕльство. Обратимся сначала к уравнению (1.27), из которого без труда получаем предельные равенства

$$
\frac{|\xi|^{2}}{\alpha} \rightarrow 2(3 \omega)^{-1}, \quad \frac{\xi^{2}}{\alpha} \rightarrow 2 i(3 \omega)^{-1}, \quad \alpha \rightarrow \infty
$$

Учитывая, далее, $(2.32)$ в (2.30), приходим к выводу, что равномерно по $\delta$ из любого ограниченного множества

$$
G(\alpha, \delta) \rightarrow G_{0}, \quad \alpha \rightarrow \infty
$$

здесь матрица $G_{0}$ имеет структуру $(2.18)$, где $\varkappa_{j}, j=1, \ldots, 4$, следует заменить на

$$
\varkappa_{1}^{0}=-\frac{1}{2}, \quad \varkappa_{2}^{0}=\frac{1}{8} i, \quad \varkappa_{3}^{0}=-\frac{1}{4}, \quad \varkappa_{4}^{0}=-\frac{1}{8} i .
$$

Таким образом, обоснование леммы сводится в первую очередь к проверке гурвицевости при всех $z>0$ предельной матрицы

$$
G_{0}+z \operatorname{diag}\{i,-i,-i, i\}
$$

Несложный подсчет с учетом (2.34) показывает, что характеристическое уравнение матрицы (2.35) после замен $4 \lambda \rightarrow \lambda, 4 z \rightarrow z$ преобразуется к виду

$$
\lambda^{4}+8 \lambda^{3}+\left(2 z^{2}+21\right) \lambda^{2}+\left(8 z^{2}+18\right) \lambda+6 z^{2}+z^{4}=0
$$

Применяя, далее, к полиному из (2.36) критерий Рауса-Гурвица, получим требуемый факт - гурвицевость матрицы (2.35) при всех $z>0$.

Обратим внимание, что при $z \ll 1$ уравнение $(2.36)$ имеет простой корень

$$
\lambda=\lambda_{0}(z), \quad \lambda_{0}(z)=-\frac{1}{3} z^{2}+O\left(z^{4}\right), \quad z \rightarrow 0
$$

а три других его корня при $z=0$ отрицательны. Поэтому при $0<z \ll 1$, $\alpha \gg 1$ гурвицевость матрицы (2.31) нуждается в отдельной проверке. Точнее говоря, проблема сводится к исследованию поведения простого ее собственного значения $\lambda_{0}(z, \alpha)$, стремяшегося к нулю при $z \rightarrow 0, \alpha \rightarrow \infty$.

Из структуры входящих в (2.31) слагаемых (см. (2.18)) вытекает вещественность коэффициентов характеристического полинома матрицы $(2.31)$, а также их четность по переменной $z$. А отсюда следует, что интересующее нас собственное значение $\lambda_{0}(z, \alpha)$ вешественно и

$$
\lambda_{0}(-z, \alpha) \equiv \lambda_{0}(z, \alpha)
$$


Как показьвает непосредственная проверка с учетом (2.18)-(2.22), фигурируюшая в $(2.24)$ матрица $\Lambda_{1}(0)$ всегда имеет нулевое собственное значение, которому отвечает собственный вектор $\operatorname{colon}(\xi, \bar{\xi},-\xi,-\bar{\xi})$. Поэтому в нашем случае автоматически выполняется равенство

$$
\lambda_{0}(0, \alpha) \equiv 0
$$

Из $(2.38),(2.39)$ следует, что тейлоровское разложение $\lambda_{0}(z, \alpha)$ по $z$ в точке $z=0$ начинается с квадратичного слагаемого, а значит, при $z=0$ является непрерывной функция $z^{-2} \lambda_{0}(z, \alpha)$. Более того, из асимптотических равенств (2.33), (2.37) заключаем, что

$$
z^{-2} \lambda_{0}(z, \alpha) \rightarrow-\frac{1}{3}, \quad z \rightarrow 0, \quad \alpha \rightarrow \infty
$$

Тем самым, собственное значение $\lambda_{0}(z, \alpha)$ отрицательно при всех $0<z \ll 1$, $\alpha \gg 1$. Лемма доказана.

Из установленной леммы следует, что для задачи $(2.29)$ в случае $\alpha \gg 1$ все условия теоремы 2 выполняются на любом фиксированном отрезке изменения параметра $\delta$. А это означает, что при подходяшем увеличении $\alpha$ и уменьшении $\varepsilon$ можно гарантировать существование у нее любого наперед заданного конечного числа устойчивых периодических решений, т.е. в рамках данной краевой задачи наблюдается феномен параметрической буферности.

Другой пример - краевая задача

$$
u_{t t}+\varepsilon u_{t}+(1+\varepsilon \alpha \cos 2 \omega t) \sin u=\varepsilon^{2} a^{2} u_{x x},\left.\quad u_{x}\right|_{x=0}=\left.u_{x}\right|_{x=\pi}=0 .
$$

В этом случае необходимость в неравенстве (1.33) отпадает по тем же причинам, что и выше. Далее, ограничения (1.30) и условие 1 здесь выполняются, поскольку $d=-3 i / 8, \varphi(z, \delta) \equiv-1 / 2$. И наконец, что характерно именно для данного примера, при всех $\delta>-\delta_{*}$, где $\delta_{*}=\sqrt{\alpha^{2} / 4-\omega^{2}}$, уравнение (1.29) имеет положительный корень

$$
z_{0}=|\xi|^{2}=\frac{8}{3}\left(\delta+\delta_{*}\right)
$$

для которого выполняется неравенство (1.31). Тем самьм, в лемме 2 и в теореме 1 в качестве промежутка изменения параметра $\delta$ здесь можно брать любой конечный отрезок

$$
\left[\delta_{1}, \delta_{2}\right] \subset\left(-\delta_{*}, \infty\right)
$$

А отсюда автоматически следует, что количество $n_{* *}(\varepsilon)$ периодических решений (1.17), (1.18) задачи (2.40), доставляемых теоремой 1 , можно неограниченно увеличивать только за счет уменьшения $\varepsilon$ и увеличения длины отрезка (2.42) (при фиксированных параметрах $\alpha, \omega)$. Для сравнения заметим, что в задаче (2.29) подобное невозможно. 
Перед проверкой условий 2 и 3 отметим, что в данном случае

$$
\varkappa_{1}=-\frac{1}{2}+\frac{i \delta}{2 \omega}-\frac{i|\xi|^{2}}{4 \omega}, \quad \varkappa_{2}=\frac{i \alpha}{4 \omega}-\frac{i \xi^{2}}{8 \omega}, \quad \varkappa_{3}=-\frac{i|\xi|^{2}}{8 \omega}, \quad \varkappa_{4}=-\frac{i \xi^{2}}{16 \omega}
$$

где $|\xi|^{2}$ определяется в (2.41), а

$$
\xi^{2}=|\xi|^{2} \frac{2\left(\delta_{*}+i \omega\right)}{\alpha}
$$

Используя $(2.43),(2.44)$ и проводя соответствующие вычисления, убеждаемся, что условие 2 эквивалентно неравенству

$$
|\xi|^{2}<8 \omega
$$

а характеристический полином матрицы

$$
\Lambda_{1}(0)+z \operatorname{diag}\{i,-i,-i, i\}, \quad z>0
$$

имеет вид

$$
\begin{aligned}
P(\lambda)= & \lambda^{4}+2 \lambda^{3}+\left(1+2 z^{2}+\frac{3 \delta_{*}|\xi|^{2}}{8 \omega^{2}}\right) \lambda+\left(2 z^{2}+\frac{3 \delta_{*}|\xi|^{2}}{8 \omega^{2}}\right) \lambda \\
& +\left(\frac{\delta_{*}|\xi|^{2}}{8 \omega^{2}}-\frac{\delta_{*}^{2}}{\omega^{2}}\right) z^{2}+z^{4} .
\end{aligned}
$$

Стандартный анализ полинома (2.47) приводит к выводу, что гурвицевость матрицы (2.46) при всех $z>0$ эквивалентна неравенству

$$
|\xi|^{2}>8 \delta_{*}
$$

Наконец, объединяя (2.45) и (2.48), убеждаемся, что условия теоремы 2 вьполняются только при

$$
2 \omega<\alpha<2 \sqrt{2} \omega
$$

и при значениях параметра $\delta$ из произвольно фиксированного отрезка

$$
\left[\delta_{1}, \delta_{2}\right] \subset\left(2 \delta_{*}, 3 \omega-\delta_{*}\right)
$$

Проделанный анализ показьвает, что в диапазоне (2.49) изменения параметра $\alpha$ буферность наблюдается и в рамках задачи (2.40). В самом деле, хотя требование $(2.50)$ не позволяет неограниченно увеличивать количество $n_{* *}(\varepsilon)$ ее устойчивых периодических решений $(1.17),(1.18)$ за счет роста $\delta_{2}-\delta_{1}$, но $n_{* *}(\varepsilon)$ может быть сделано сколь угодно большим при $\omega \rightarrow 1, \varepsilon \rightarrow 0$ или при $a \rightarrow 0$ (см. замечания после теоремы 1 ).

Подводя итог, отметим, что в то время как в случае задачи (2.29) буферность представляет собой основную особенность динамики, реализующуюся в широком диапазоне изменения параметров $\alpha, \omega$, то в случае задачи (2.40) ситуация иная: 
теоремы 1 и 2 дают весьма скудную информацию о характере ее устойчивых колебаний. Действительно, как уже говорилось выше, при фиксированных $\alpha, \omega$ количество периодических решений задачи (2.40), доставляемых теоремой 1 , асимптотически велико, т.е. стремится к бесконечности при $\varepsilon \rightarrow 0$. Однако устойчивыми среди них являются лишь те решения (1.17), для которых параметр $\delta_{n}(\varepsilon)($ см. $(1.5))$ принадлежит отрезку (2.50). Количество же последних асимптотически конечно (ограничено при $\varepsilon \rightarrow 0$ ). Таким образом, даже при выполнении неравенств (2.49) подавляюшее большинство периодических решений задачи (2.40) неустойчиво, а при $\alpha>2 \sqrt{2} \omega$ неустойчивыми становятся они все. В последнем случае вопрос об аттракторах остается открытьм.

В заключение считаем своим долгом особо подчеркнуть, что по тематике данная статья является естественным продолжением начатых Ю. С. Колесовым исследований особенностей динамики, вызванных параметрическим внешним воздействием в случае нелинейных телеграфных уравнений (см. [8], [10]). Однако в отличие от работ [8], [10], где инструментом анализа был предложенный Ю. С. Колесовым метод квазинормальных форм, нами используется специальная техника изучения высокомодовых колебаний, развитая в [9], [13].

\section{Список литературы}

1. Боголюбов Н. Н., Митропольский Ю. А. Асимптотические методы в теории нелинейных колебаний. М.: Наука, 1974.

2. Митропольский Ю. А., Мосеенков Б. И. Асимптотические решения уравнений в частных производных. Киев: Вища школа, 1976.

3. Якубович В. А., Старжинский В. М. Линейные дифференциальнте уравнения с периодическими коэффициентами и их приложения. М.: Наука, 1972.

4. Фомин B. Н. Математическая теория параметрического резонанса в линейных распределенных системах. Л.: Изд-во ЛГУ, 1972.

5. Колесов А.Ю., Колесов Ю.С. Принцип сведения Боголюбова-Митропольского в задаче о параметрическом возбуждении автоволн // Докл. АН СССР. 1989. Т. 307. № 4. C. $837-840$.

6. Колесов Ю.С. Нелинейный параметрический резонанс в сингулярно возмущенном телеграфном уравнении // Дифференц. уравнения. 1991. Т. 27. № 10. С. 1828-1829.

7. Колесов А. Ю. Параметрические колебания решений телеграбоног уравнения с умеренно малой диффузией // Сиб. матем. журн. 1992. Т. 33. №6. С. 79-86.

8. Колесов Ю.С. Асимптотика и устойчивость нелинейных параметрических колебаний сингулярно возмущенного телеграффного уравнения // Матем. сб. 1995. Т. 186. № 10. C. $57-72$.

9. Колесов А.Ю., Мищенко Е.Ф., Розов Н.Х. Явление параметрической буферности в системах параболических и гиперболических уравнений с малой диффузией $/ / \mathrm{У}_{\text {кр. }}$ матем. журн. 1998. Т. 50. № 1. С. 22-35.

10. Колесов Ю.С. Параметрические колебания сингулярно возмущенного телеграфного уравнения с маятниковой нелинейностью // Матем. сб. 1998. Т. 189. № 3. С. 69-82.

11. Колесов Ю. С., Майоров В. В. Новый метод исследования устойчивости решений линейных дифференциальных уравнений с близкими к постоянњым почти периодическими коэффициентами // Дифференц. уравнения. 1974. Т. 10. № 10. С. 1778-1788.

12. Кубыикин Е. П. Параметрический резонанс в линейных периодических системах с последействием // Исследования по устойчивости и теории колебаний. Ярославль: Изд-во ЯГУ, 1978. С. 43-76. 
13. Камбулов В. Ф., Колесов А. Ю., Розов Н.X. Существование и устойчивость быстро осциллирующих циклов у нелинейного телеграфного уравнения // ЖВМ и МФ. 1998. T. 38. № 8 . С. 1287-1300.

14. Колесов А. Ю., Мищенко Е.Ф., Розов Н.X. Асимптотические методы исследования периодических решений нелинейных гиперболических уравнений. М.: МАИК "Наука", 1998. (Труды МИАН. 1998. Т. 222.).

15. Васильева А.Б., Кащенко С.А., Колесов Ю.С., Розов Н.Х. Бифуркация автоколебаний нелинейных параболических уравнений с малой диффузией // Матем. сб. 1986. T. 130 (172). № 4 . C. 488-499.

Ярославский государственный университет им. П. Г. Демидова;

Московский государственньй университет им. М.В. Ломоносова
Поступила в редакцию

08.12.1999 